\title{
Regulación y poder empresarial: el caso del transporte público en Santiago (2007-2017)
}

\author{
Antoine Maillet 1 \\ Sebastián Carrasco ${ }^{2}$ \\ 1 Universidad de Chile / Instituto de Asuntos Públicos, Santiago, Chile \\ 2 Universidad Mayor, Santiago, Chile
}

Una plena comprensión de los procesos regulatorios requiere tomar en cuenta su dimensión política, hasta ahora escasamente considerada en la literatura de administración pública. A este objetivo contribuye el concepto de poder empresarial que ha desarrollado la economía política. Como lo demuestra el estudio de caso de Transantiago, resulta de especial interés la vertiente estructural del poder empresarial. El process tracing aplicado al caso de este sistema de transporte demuestra que la incapacidad de las autoridades de imponer sus preferencias se debe principalmente a las restricciones estructurales que enfrentan, cuyos efectos se manifiestan de manera distinta en la etapa de adjudicación de la licitación y en la fase de implementación.

Palabras-clave: regulación; proceso regulatorio; concesión; poder empresarial; transporte público.

\section{Regulação e poder de negócios: 0 caso do transporte público em Santiago (2007-2017)}

O pleno entendimento dos processos regulatórios exige considerar sua dimensão política, até agora raramente considerada na literatura da administração pública. O conceito de poder de negócio desenvolvido pela economia política contribui para esse objetivo. Como demonstra o estudo de caso do Transantiago, o poder estrutural dos negócios é de especial interesse. O process tracing aplicado ao caso desse sistema de transporte, inaugurado em 2007, mostra que a incapacidade das autoridades em impor suas preferências se deve principalmente às restrições estruturais que enfrentam, cujos efeitos variam na fase de leilão e implementação do processo de regulação.

Palavras-chave: regulação; processo regulatório; concessão; poder de negócio; transporte público.

\section{Regulation and business power: the case of public transportation in Santiago (2007-2017)}

Full understanding of regulatory processes requires taking into account their political dimension, until now rarely considered in public administration literature. The concept of 'business power' developed in comparative political economy contributes to comprehend this dimension. The case study of the transport system "Transantiago" presented in this article demonstrates that businesses' structural power is of special interest. The process tracing applied to the case of Transantiago, shows that the inability of the authorities to impose their preferences is mainly due to the structural constraints they faced. The effects of this inability are observed in the bidding process and in the phase of implementation of the regulation process.

Keywords: regulation; regulatory process; concession; business power; public transportation. 


\section{INTRODUCCIÓN}

Si bien con el desarrollo del concepto de gobernanza el estudio de la regulación ha ido considerando una variedad mayor de actores (Baldwin, Cave, \& Lodge, 2010), desde la administración pública se mantiene un foco predominante en las acciones de las autoridades, en particular en los diseños adecuados para lograr un proceso regulatorio de calidad (Engel, Fisher, \& Galetovic, 2010), y en los riesgos de comportamientos oportunistas de los gobiernos (Bitrán, Nieto-Parra, \& Robledo, 2013). Lo simétrico de este sesgo hacia una especie de unilateralidad del poder de la autoridad es el repudio de la captura. Bajo esta óptica, toda regulación hecha en concertación o bajo presión de actores económicos sería capturada. Sin duda, en un proceso de regulación, las relaciones entre las autoridades políticas y ciertos grupos económicos están expuestas a que los primeros sean capturados por los segundos (Stigler, 1971). Pero el Estado puede también establecer una relación virtuosa con los actores privados, de forma tal que genere una mayor eficacia en la provisión de servicios públicos y que, a la vez, le permita mantener su autonomía (Evans, 1996). Aun reconociendo la importancia de los actores formalmente a cargo del diseño de la regulación, de modo general, la complejidad de la toma de decisiones en la esfera pública vuelve necesaria la incorporación de otros actores en los análisis empíricos que se hagan de ésta (Dente \& Subirats, 2014). Para dar cuenta de los fuertes constreñimientos que limitan las decisiones soberanas de la autoridad en los procesos regulatorios, este artículo invita a considerar el concepto desarrollado en economía política de poder empresarial (Hacker \& Pierson, 2002), que contribuye a una mejor comprensión de la dimensión política de la regulación, y en particular de mecanismos de licitación.

Esta reflexión es arraigada empíricamente en un proceso regulatorio paradigmático a la escala continental, el del transporte público en Santiago, capital de Chile. En febrero de 2007 se puso en marcha el sistema Transantiago, que consistió en la creación de un nuevo mercado regulado del transporte público (Ureta, 2015). Fueron licitadas distintas unidades de negocio, quedando a cargo de ellas empresas nacionales y extranjeras (Maillet, 2015). Los numerosos problemas operativos, que rápidamente derivaron en dificultades políticas (Mardones, 2008; Olavarría, 2013), abrieron una continua disputa entre los operadores de transporte y las autoridades - principalmente el Ministro de Transporte y Telecomunicaciones - teniendo como foco principal los contratos, sus disposiciones y la forma de monitorear su cumplimiento. Desde la crisis del inicio, el año 2007, hasta la fallida nueva licitación de 2018, los sucesivos ministros han tenido la voluntad de modificar o incluso caducar los contratos de concesión inicialmente firmados. Sin embargo, ni la exposición permanente del tema en los medios de comunicación, ni el repudio transversal al sistema han sido suficientes para revertir completamente los problemas de operación. Ante esta situación, ¿ cómo lograron los operadores de Transantiago resistir esta presión? En términos más conceptuales, ¿cómo explicar las dificultades de las autoridades en imponer sus preferencias en el proceso regulatorio?

Una posible respuesta a esta interrogante empírica y su corolario teórico puede venir desde la economía política, en particular del concepto de poder empresarial - o business power - , que se refiere a la influencia que ejercen los actores empresariales en el proceso político (Culpepper, 2011; Hacker \& Pierson, 2002), la cual se puede expresar de dos modos genéricos. Uno - llamado poder 
instrumental - consiste en la presión organizada de los actores económicos sobre la política; y el otro, conocido como poder estructural, se manifiesta a través del peso propiamente económico de los actores empresariales, en particular el riesgo que detengan las inversiones en caso de preocupación por las políticas implementadas (Fairfield, 2015a). A pesar de la capacidad explicativa que posee esta literatura, aún no ha sido considerada en los estudios de administración pública y política pública. Por eso el objetivo de este artículo es demostrar, a partir del estudio de caso del Transantiago, la contribución analítica al estudio de los procesos de regulación que puede realizar esta herramienta conceptual.

En función de esta pregunta, la principal hipótesis de investigación es que el poder empresarial que ejercen los operadores juega un rol fundamental en que la autoridad política no pueda imponer sus preferencias en el proceso regulatorio. Por este término se entiende la relación que se establece entre actores públicos y privados a través de la licitación de un servicio, incluyendo así tanto el diseño de procesos competitivos para la adjudicación de contratos, como la implementación de aquellos. Para desarrollar esta hipótesis, a continuación presentamos el marco teórico del estudio. Luego se plantea el estudio de caso, considerando hipótesis alternativas. Todas estas están evaluadas a través del process tracing del caso. Finalmente, en la conclusión se señalan las implicancias más generales de los resultados de este estudio.

\section{INCORPORAR EL BUSINESS POWER PARA DESARROLLAR LA DIMENSIÓN POLÍTICA DEL PROCESO REGULATORIO: HACIA UNA MIRADA MÁS COMPLEJA}

\subsection{Regulación y contractualización: la visión tradicional y sus vacíos}

En primer lugar, es necesario definir lo que se entiende por regulación en este artículo. Tradicionalmente, se ha considerado como tal "[...] el control sostenido y focalizado ejercido por una autoridad pública sobre actividades valoradas por la comunidad" (Selznick, 1985, como citado en Baldwin et al., 2010, p. 12), pero su definición se ha extendido hacia "[...] el uso intencional de autoridad para afectar el comportamiento de un actor de acuerdo a ciertos estándares, mediante instrumentos de recopilación de información y modificación de comportamiento" (Black, 2001, como citado en Baldwin et al., 2010, p. 12). Como se aprecia, la definición tradicional tenía un foco muy restringido, sobre el control, lo que implicaba un número reducido de instrumentos de política. Con la definición actualizada, pasan a formar parte de la regulación numerosos instrumentos basados en incentivos, en un cambio de lógica que ha sido considerado como una transición para el Estado desde el "remar" (rowing) hacia el "llevar el timón" (steering) (Osborne \& Gaebler, 1992). De esto último participan todos los mecanismos de delegación y contractualización, como las licitaciones que son el objeto de estudio de este artículo, y las posteriores renegociaciones.

De manera general, el principal mecanismo utilizado para formalizar estas relaciones entre Estado y privados corresponde a la contratación pública (Bhagat, 2017; Mukhopadhyay, 2011). En Chile, estos contratos recaen en la figura de la licitación, que corresponde al procedimiento mediante el cual un privado concursa para proveer un servicio o bien público. La licitación se ha vuelto un instrumento común en la introducción de competencia en la regulación o adquisición de bienes y servicio ofertados por privados, que tiene lugar cuando existe un solo comprador (el sector público) 
y múltiples oferentes, ya sea en contextos de competencia imperfecta o monopolios naturales; así como en industrias reguladas, o en mercados pequeños y con información imperfecta (Menezes, Pitchford, \& Wait, 2003). No obstante, en general las licitaciones tienen dificultades para generar competencia. Esta es una operación difícil, en donde existen potenciales problemas en la relación posterior. Empero, en un afán normativo, la literatura también ha tendido a establecer el diseño óptimo de un contrato. Autores como Engel et al. (2010) señalan la importancia de equilibrar el riesgo de demanda, las distorsiones tarifarias y el costo de oportunidad de los fondos públicos, generando una garantía mínima y máxima de ingresos. La gran dificultad que se debe sortear en el diseño de un contrato es fijar el nivel de flexibilidad de este. Esta flexibilidad oscila entre diseños muy flexibles, que pueden dar pie a renegociaciones oportunistas, o diseños rígidos, los cuales pueden producir la pérdida de oportunidades para renegociar en miras al interés público, siendo fundamental alcanzar un nivel adecuado entre los dos extremos (Athias \& Saussier, 2007).

No obstante los aspectos técnicos señalados anteriormente, tanto las licitaciones como la renegociación de los contratos constituyen procesos donde las variables políticas juegan un rol fundamental (Bitrán et. al, 2013), pero que no son mayormente abordadas bajo estos enfoques. La literatura reconoce, por cierto, que existe una tensión entre los intereses de los privados y la necesidad de crear valor público del sector gubernamental, lo que configura múltiples tipos de alianzas públicoprivadas (Bovaird, 2006; Wettenhall, 2003), pero no ha explorado de manera profusa las relaciones conflictivas que las subyacen.

Una aproximación solo desde la economía o la gestión pública para comprender la dinámica de los contratos tiene por lo tanto claros limitantes. Por ejemplo, la evidencia muestra que, pese a que los agentes gubernamentales puedan contar con poderosas herramientas para hacer cumplir la ejecución de los contratos, sobre todo en lo que refiere a la imposición de sanciones, estas en general no son usadas (Girth, 2012). En tal sentido, siendo factible técnicamente y viable legalmente, las obligaciones contractuales no se hacen cumplir, por lo que abordar la dinámica de la contractualización desde otra mirada analítica, distinta a las corrientes predominantes, es la contribución original que hace este trabajo.

\subsection{Hacia una mirada política: contribución del business power al estudio de las dinámicas del proceso regulatorio}

En el marco de las políticas regulatorias, las agencias regulatorias nunca serán completamente autónomas, o estarán totalmente capturadas, sino que su comportamiento va a estar determinado por las interacciones que tengan con otros actores sociales (Gilardi \& Maggetti, 2011). Por lo tanto, para entender el proceso regulatorio se requiere el desarrollo de una mirada que ponga de relieve la dimensión política de procesos plagados de conflictividad, incorporando otros actores en el análisis; especialmente los actores empresariales, por ser los entes regulados en la regulación. En este sentido, la economía política entrega poderosas herramientas analíticas. En particular, la literatura referente al business power se vuelve fundamental para explicar este tipo de dinámicas conflictuales entre actores públicos y empresariales. Los elementos específicos de esta perspectiva teórica se detallan a continuación.

En la literatura sobre business power, el empresariado puede ejercer su influencia política a través de dos principales tipos de poder. Por una parte, se encuentra el poder estructural, el cual se refiere a la amenaza creíble que perciben los policy-makers de que una reforma en el ámbito político reducirá la inversión privada, teniendo efectos negativos sobre los agregados económicos (Fairfield, 2015a). 
En base a esto, el ámbito de lo estructural viene dado por la posición económica privilegiada que los agentes del sector privado ocupan en los sistemas capitalistas (Fairfield, 2015a). Si bien es complejo establecer cómo opera este poder en la arena política (Culpepper, 2015), es relevante señalar que está muy ligado al peso relativo que el empresariado de un sector económico específico posea, en relación con el rol que el Estado tenga en la economía (Fairfield, 2015a). En la medida en que el Estado sea un actor central en la economía, el poder estructural será débil. Sin embargo, en las economías capitalistas, como es bien conocido, son las empresas las que producen bienes, crean empleos y deciden cuándo, dónde y cómo invertir (Lindblom, 1982), lo que hace del poder estructural una amenaza permanente en este tipo de sistemas. En tal sentido, en el ejercicio del poder estructural son centrales las percepciones y expectativas que tienen los policy-makers respecto a la reacción de los empresarios ante determinadas decisiones (Bell \& Hindmoor, 2013).

Por lo tanto, en general la literatura referida al poder estructural establece como preocupación central de los tomadores de decisión la amenaza que representan las políticas públicas para la inversión de las empresas (Fairfield, 2015a). En el marco de las actividades regulatorias, en donde las agencias autónomas regulan las actividades empresariales (Jordana \& Levi-Faur, 2005), interviniendo en la esfera económica para dirigir la acción privada hacia la realización de objetivos públicos (Christensen \& Lægreid, 2006), el Estado se ve enfrentado a una tensión entre la necesidad de regular a los entes privados, a la vez que busca no interferir con la inversión de estos para la provisión de servicios que son valorados por la comunidad. Esta perspectiva analítica es especialmente relevante para analizar el caso del Transantiago. La necesidad de atraer inversión privada para lograr poner en marcha el servicio puede significar que las reglas institucionales establecidas no sean lo suficientemente exigentes para las empresas como pudiesen ser, asegurando así el correcto funcionamiento del servicio. En el marco de un proceso licitatorio, esto significa establecer incentivos en los contratos para atraer oferentes que participen de dicha licitación.

Así, el poder estructural incide especialmente en los procesos de formulación de políticas, donde las decisiones que se toman están constreñidas por la necesidad de incentivar la inversión de los actores privados (Fairfield, 2015a). Sin embargo, a diferencia de lo establecido por la literatura tradicional sobre poder empresarial, proponemos que en el caso de las políticas regulatorias el poder estructural no se expresa exclusivamente en los procesos de formulación de las políticas, donde existe la necesidad de mantener y/o atraer inversión privada. Al contrario, consideramos que, en la fase de implementación, cuando las autoridades ejecutan los instrumentos regulatorios contra las empresas también es posible observar dicho poder estructural, pero manifestándose de una manera distinta. En la fase de implementación, la amenaza de la quiebra de las firmas, y la consecuente paralización en la entrega del servicio que eso significa, puede generar que los instrumentos regulatorios no se apliquen con el rigor legalmente permitido. En definitiva, las decisiones de las autoridades no solo se ven constreñidas por la necesidad de resguardar la inversión, sino que también porque estas firmas no dejen de operar. En el caso bajo estudio, el riesgo de discontinuar el servicio de transportes generaría que las autoridades apliquen las disposiciones contractuales de manera cauta en contra las empresas. Esto constituye una forma de poder estructural original, propio de los procesos regulatorios, que debería tener mayor consideración por parte de quienes se interesan en estos fenómenos.

En contraste con el poder estructural, el poder instrumental proviene de recursos políticos que son construidos deliberadamente para influenciar las políticas públicas de una manera mucho más efectiva (Fairfield, 2015a). Estos recursos pueden expresarse de distintas formas. Entre los instrumentos que utilizan para incidir en las políticas se encuentran los vínculos que establecen los grupos empresariales 
con los partidos políticos; la consulta institucionalizada, que se expresa principalmente en reuniones formales con la autoridad y mesas de negociación; el reclutamiento de representantes de los empresarios en cargos gubernamentales; vínculos informales de distinto tipo, como los familiares y profesionales; la cohesión de los grupos empresariales, para actuar de manera coordinada frente a la autoridad (Álamos, 2017). La experticia técnica, el acceso a los medios y el dinero (con el que se pueden financiar campañas políticas, por ejemplo) completan esta lista (Fairfield, 2015a). En el caso de la regulación, estos mecanismos se pueden utilizar para incidir en las decisiones del regulador. Específicamente, las empresas desarrollan distintas estrategias dependiendo del ambiente regulatorio existente (Henson \& Hooker, 2001, como citado en Gilardi \& Maggetti, 2011). De ahí la relevancia de comprender las acciones que emprendan las empresas en base al poder instrumental que ostenten. En el caso de transportes, implicaría buscar controlar los contratos y liderar los procesos de renegociación, para que así la política regulatoria se corresponda con las preferencias que tienen las empresas.

En contraposición a la perspectiva del business power, otra de las hipótesis alternativas de la investigación hace referencia a las debilidades institucionales de la autoridad como factor explicativo de los contratos y las decisiones. De manera general, esta perspectiva nos remite teóricamente al concepto de capacidad estatal. Así, baja capacidad estatal llevaría a debilidades institucionales. Desde la literatura más clásica en la materia, la capacidad estatal se expresa cuando las instituciones públicas logran llevar a cabo sus objetivos, especialmente existiendo oposición de sectores de la sociedad civil (Sckocpol, 1985). En tal sentido, las elecciones de política pública en materia de regulación de los mercados se encontrarían afectadas por factores endógenos relativos a la capacidad estatal (Besley \& Persson, 2009). Sintetizando, dicha capacidad se encontraría expresada principalmente en los elementos coercitivos, fiscales y administrativos del gobierno (Cingolani, 2013). Empíricamente, estas dimensiones pueden ser expresadas en las habilidades técnico-políticas de las autoridades políticas para hacer cumplir sus deseos (Centeno, 2002), el grado de profesionalización de la burocracia y su independencia del poder político para la toma de decisiones en los procesos de implementación de políticas (Peters, 1996) y la habilidad de recaudar impuestos, con el consiguiente uso de esos recursos para proveer bienes públicos y hacer transferencias (Besley \& Persson, 2008), en función de los objetivos definidos.

Estos distintos conceptos - operacionalizados en la sección siguiente - constituyen el marco analítico del estudio de caso desarrollado a continuación.

\section{DISEÑO DEL ESTUDIO DE CASO}

Desde su comienzo hasta la fecha, el Transantiago ha tenido numerosas modificaciones con el fin de mejorar el servicio de transportes. Esto ha provocado que el gobierno no haya logrado conseguir completamente los objetivos que se ha planteado de tener un " [...] sistema de transporte [...] mejor que cualquier otro país" (coordinador de Transantiago, citado en Maillet, 2008). Para explicar por qué el gobierno no ha podido imponer sus preferencias, esta investigación plantea la realización de un estudio de caso, mediante la metodología de process tracing (Bennett \& Checkel, 2015; Bril, Maillet, \& Mayaux, 2017; Trampusch \& Palier, 2016), la cual nos permite establecer relaciones causales entre distintas variables, en función de las hipótesis planteadas.

En tal sentido, la principal hipótesis de investigación es que el poder empresarial que ejercen los empresarios juega un rol fundamental en que la autoridad política no pueda imponer sus preferencias sobre estos operadores. Desde esa mirada, se distinguen las estrategias de poder instrumental y el poder 
estructural que se ejercen por parte de las empresas, en el marco de esta política sectorial. La hipótesis alternativa que se desarrolla para explicar la incapacidad de las autoridades para imponer sus preferencias considera posibles deficiencias de la autoridad misma. Ciertas características de los organismos públicos y las autoridades podrían ser las causantes de que estos actores no logren implementar sus decisiones de acuerdo con sus preferencias para el transporte público de la capital. Cuestiones como la debilidad política, la falta de capacidad e idoneidad técnica y los constreñimientos presupuestarios serían las principales expresiones de esta deficiencia. Como suelen permitir los estudios de caso, la respuesta no consiste en dirimir entre las hipótesis, sino que, en una lógica de causalidad compleja, se trata de encontrar y jerarquizar los factores determinantes en el resultado de interés (Goertz \& Mahoney, 2012).

La construcción de una narrativa donde se testea estas hipótesis de investigación se realizó con base en un material empírico profuso y diverso. Junto a un equipo de investigación ${ }^{1}$, se sistematizaron 761 artículos de prensa del Diario Financiero, un periódico de circulación nacional especializado en abordar temas económicos y regulatorios. Los artículos seleccionados representan todas aquellas entradas en el periódico que contengan el término "Transantiago", y que han aparecido en este medio entre 2008 y 2018. Además, se realizaron 16 entrevistas a actores claves del sector público y privado, así como dos foros públicos con expertos de diversas disciplinas ${ }^{2}$. Entre los entrevistados se encuentran ex ministros, subsecretarios, altos directivos públicos, empresarios transportistas y expertos en transportes, pertenecientes a distintas universidades nacionales. La información así recolectada fue complementada con otras fuentes de prensa, documentos oficiales del gobierno, investigaciones periodísticas, informes técnicos, leyes y las discusiones de los proyectos de ley que se desarrollaron en el Congreso Nacional.

El cuadro a continuación sintetiza las hipótesis y precisa las implicancias observables (Bril et al., 2017) para cada una.

\section{CUADRO 1 HIPÓTESIS, OPERACIONALIZACIÓN E IMPLICANCIAS OBSERVABLES}

\begin{tabular}{|c|c|c|}
\hline Hipótesis & 0peracionalización ${ }^{3}$ & Implicancias observables \\
\hline \multirow[t]{4}{*}{$\begin{array}{l}\text { H1a. Poder instrumental de } \\
\text { los operadores. }\end{array}$} & Cohesión & $\begin{array}{l}\text { La colaboración entre los operadores los fortalece } \\
\text { frente a la autoridad. }\end{array}$ \\
\hline & Experticia técnica & $\begin{array}{l}\text { Los operadores entregan experticia técnica a los } \\
\text { organismos públicos, haciéndolos dependientes. }\end{array}$ \\
\hline & $\begin{array}{l}\text { Acceso a los medios de } \\
\text { comunicación }\end{array}$ & $\begin{array}{l}\text { Los operadores privados gozan de una buena imagen } \\
\text { pública. }\end{array}$ \\
\hline & $\begin{array}{l}\text { Vínculos informales con } \\
\text { autoridades y partidos - Puerta } \\
\text { giratoria }\end{array}$ & $\begin{array}{l}\text { Actores del sector privado y público comparten redes, } \\
\text { personales, profesionales y-o políticas que permiten a } \\
\text { los primeros influir sobre los segundos. }\end{array}$ \\
\hline
\end{tabular}

\footnotetext{
${ }^{1}$ Este equipo está compuesto por Alessandra Altamirano, Camila Flores, Pablo Mancilla y Matías Silva, todos estudiantes de la carrera de administración pública de la Universidad de Chile.

2 “Neoliberalismo híbrido y políticas públicas. ¿Cómo se expresa en el Transantiago?”, Escuela de Gobierno y Gestión Pública, INAP, Universidad de Chile, 17-10-2017; y “Nueva licitación de Transantiago. ¿De dónde venimos y hacia dónde vamos?”, INAP, Universidad de Chile, 26-01-2018.
} 


\begin{tabular}{|c|c|c|}
\hline Hipótesis & Operacionalización ${ }^{3}$ & Implicancias observables \\
\hline \multirow[t]{2}{*}{$\begin{array}{l}\text { H1b. Poder estructural de los } \\
\text { operadores. }\end{array}$} & Suspensión de futuras inversiones & $\begin{array}{l}\text { Los empresarios amenazan (o existe la percepción por } \\
\text { parte de la autoridad) con abandonar la actividad. }\end{array}$ \\
\hline & Riesgos de quiebra & $\begin{array}{l}\text { Las empresas están en riesgo de quiebra por las } \\
\text { disposiciones contractuales, lo que amenazaría la } \\
\text { continuidad del servicio. }\end{array}$ \\
\hline \multirow{3}{*}{$\begin{array}{l}\text { H2. Deficiencias de los } \\
\text { organismos públicos y la } \\
\text { autoridad }\end{array}$} & $\begin{array}{l}\text { Falta de capacidad/idoneidad } \\
\text { técnica, }\end{array}$ & $\begin{array}{l}\text { El sector público no dispone de los conocimientos } \\
\text { expertos suficientes para dirigir el proceso regulatorio. }\end{array}$ \\
\hline & Debilidad política & $\begin{array}{l}\text { La autoridad de turno es percibida como débil por los } \\
\text { otros actores. }\end{array}$ \\
\hline & Constreñimientos presupuestarios. & $\begin{array}{l}\text { La estrechez de presupuesto limita los márgenes de } \\
\text { negociación del sector público. }\end{array}$ \\
\hline
\end{tabular}

Fuente: Elaborado por los autores.

Para testear empíricamente estas hipótesis, se analiza la trayectoria de la política de transporte público en Santiago a lo largo de diez años, dando cuenta de los principales hitos de la relación entre autoridades y empresas transportistas en el marco de la regulación del Transantiago.

\section{DE CONFLICTOS Y RENEGOCIACIONES: DIEZ AÑOS DE TRANSANTIAGO}

\subsection{Adjudicación e inicios del Transantiago: la sombra del poder estructural}

El Transantiago buscaba brindar un sistema de transporte público moderno, sostenible e integrado para toda la ciudad, estructurandóse a través del instrumento de las licitaciones (Figueroa \& Forray, 2011). Sin embargo, desde su formulación se observa que la preocupación central de los diseñadores del sistema de transportes capitalino está en lograr la inversión de las firmas privadas. La necesidad de atraer suficientes inversores en el ámbito de transporte genera que los tomadores de decisión establezcan medidas en los contratos del proceso licitatorio que vayan en directo beneficio de los empresarios. Así buscaban asegurar que hubiera suficientes competidores, para que el sistema pudiese operar. Concretamente, a fines de 2003 se llevó a cabo la presentación de las Bases de Licitación de Uso de Vías, en donde se buscó ante todo entregar seguridad a los inversionistas, descuidando aspectos en la prestación de servicios (Espacio Público, 2017). Esta cuestión luego se expresaría en distintas deficiencias en la entrega del servicio. Estos elementos pueden observarse en declaraciones hechas por un ex ministro de transportes:

“[...] el contrato original, era un contrato que estaba pensado para atraer inversionistas. Y no estaba pensado en los pasajeros, o sea, el sistema tenía tanto rechazo cuando se diseñó que el mundo de las empresas no quería meterse, sentían que se estaban metiendo en una cosa que estaba mal diseñada" (Entrevista con ex ministro no 2).

\footnotetext{
${ }^{3}$ No se consideró "consulta institucionalizada", por no tener ninguna instancia de aquello en el sector del transporte en Chile.
} 
Así, aparece en esta declaración una primera arista del poder estructural que tienen las empresas en la relación de regulación. La formulación y adjudicación misma del contrato estaba constreñida por el riesgo de tener una licitación desierta. Para evitar esto, desde un inicio se tuvo que diseñar un contrato atractivo para "los inversionistas". Por lo tanto, el poder estructural incide fuertemente en este primer proceso de adjudicación. Teóricamente, acá se logra observar la operación del poder estructural en sus términos más clásicos. Vale decir, desde un primer momento las autoridades están preocupadas por generar una inversión de los actores privados (Hacker \& Pierson, 2002) que intuyen podría ser escasa. En consecuencia, diseñan los instrumentos de la política regulatoria en función de estos elementos.

Al momento de la adjudicación en el año 2004, fueron 10 las empresas que firmaron los contratos, teniendo entre sus novedades el ingreso de inversionistas colombianos, quienes se adjudicaron uno de los cinco contratos troncales, mientras que las demás unidades de negocio fueron adquiridas por operadores nacionales pertenecientes al antiguo sistema de transportes (Gómez-Lobo, 2007).

\subsection{El poder estructural en la regulación y el margen acotado de las autoridades transportistas (2007-2014)}

Los primeros días del Transantiago fueron caóticos, provocando un fuerte impacto económico y político en el país (Mardones, 2008). Por lo mismo, la solicitud de renuncia al entonces ministro Sergio Espejo no sorprendió mayormente, pues el Transantiago venía acarreando diversas dificultades en su funcionamiento. Sin embargo, todos los ministros de transportes a partir de esta etapa han ostentado importantes credenciales académicas, a la vez que dan cuenta de una gran experiencia en el sector público, privado y en el mundo académico. Claramente no se trata de autoridades débiles. El siguiente cuadro resume las características de estos relevantes actores políticos.

\section{CUADRO 2 MINISTROS DE TRANSPORTES EN CHILE (2007-2018)}

\begin{tabular}{|c|c|c|c|c|c|}
\hline Ministro & Profesión & $\begin{array}{c}\text { Grado } \\
\text { académico }\end{array}$ & \multicolumn{2}{|c|}{ Período como ministro } & Trayectoria antes de ser ministro \\
\hline René Cortázar & $\begin{array}{l}\text { Ingeniero } \\
\text { Comercial }\end{array}$ & Doctor & 27-03-2007 & $10-03-2010$ & Ministro; director de diversas empresas \\
\hline Felipe Morandé & $\begin{array}{l}\text { Ingeniero } \\
\text { Comercial }\end{array}$ & Doctor & $11-03-2010$ & $16-01-2011$ & $\begin{array}{c}\text { Académico; consultor de } \\
\text { organizaciones internacionales; } \\
\text { economista jefe del banco central }\end{array}$ \\
\hline $\begin{array}{l}\text { Pedro Pablo } \\
\text { Errazuriz }\end{array}$ & Ingeniero Civil & Magíster & $17-01-2011$ & $10-03-2014$ & $\begin{array}{l}\text { Gerente de distintas empresas y de } \\
\text { empresas de transportes (Lan) }\end{array}$ \\
\hline $\begin{array}{l}\text { Andrés Gómez- } \\
\text { Lobo }\end{array}$ & $\begin{array}{l}\text { Ingeniero } \\
\text { Comercial }\end{array}$ & Doctor & $11-03-2014$ & 13-03-2017 & $\begin{array}{l}\text { Investigador; asesor; consultor de } \\
\text { organizaciones internacionales y } \\
\text { director de la empresa pública Metro }\end{array}$ \\
\hline Paola Tapia & Abogado & Magíster & 14-03-2017 & $11-03-2018$ & $\begin{array}{l}\text { Altos cargos en la administración } \\
\text { pública; asesora ministerial }\end{array}$ \\
\hline
\end{tabular}

Fuente: Elaborado por los autores. 
Asume entonces René Cortázar, a quien se le encarga solucionar la situación con plenos poderes y respaldo transversal. Ocupa un rol fundamental en las primeras instancias de renegociación de contratos. La importante capacidad técnico-política para llevar adelante distintos procesos políticos, sumado a las potestades entregadas por la Presidenta, le conferían a Cortázar una alta capacidad en términos coercitivos (Cingolani, 2013).

Al momento de su llegada, las deficiencias en la operación eran muy graves, y en buena parte atribuibles al oportunismo de operadores permitido por los contratos, por lo cual su renegociación era una prioridad. En primera instancia, la renegociación de contratos estuvo enfocada en atender problemáticas relacionadas al número de buses en las calles, la reducción de la evasión, y la atención de los usuarios, ya que los dueños de las líneas concesionadas gozaban de condiciones que les permitían utilidades sin cumplir con la cantidad de buses requerida, y con multas que tendían a ser insignificantes (Ramírez \& Minay, 2007). Para cumplir con sus objetivos, Cortázar combinó incentivos ("zanahoria") con presión ("garrote") para relacionarse con las empresas del Transantiago, adquiriendo así una actitud más confrontacional. Como él mismo escribió después:

"Zanahorias" ya no había, [...] sólo quedaba el "Garrote", que consistía en utilizar la capacidad del Estado para caducar la concesión de algún operador que no estuviera cumpliendo de forma adecuada con su contrato" (Cortázar, 2015, p. 73).

La caracterización realizada en los párrafos precedentes parece contradecir lo expresado en la literatura sobre business power. Típicamente, se ha descrito a la elite empresarial chilena como un grupo que ha desarrollado importantes fuentes de poder instrumental (Fairfield, 2015b). Sin embargo, las características contextuales específicas que se vislumbran por esa época dificultan el uso del poder instrumental. El repudio general al sistema por parte de los usuarios dejaba pocas oportunidades a las empresas para que se presentaran como las víctimas de un abuso estatal. Aun así, el proceso fue "[...] una cuestión larga, porque las empresas se corrían de la negociación, las empresas no querían negociar" (Entrevista con dirigente empresarial no 2), mientras que el gobierno insistía en que las grandes fallas del sistema eran los contratos.

En este contexto, el ministro encontró una forma de ejercer presión con la caducación en el año 2008 del contrato para la zona G, a cargo de la empresa Buses Gran Santiago. En este caso, el uso del "garrote" afectó a una empresa pequeña, que operaba en torno a 200 buses, a comparar con los 5000 del sistema. Algunas dinámicas similares luego se observarían durante la presidencia de Sebastián Piñera (2010-2014). El primer ministro de Transportes de este período, Felipe Morandé, caducó un contrato con una de las empresas operadoras del Transantiago, Transaraucaria, por incumplimiento de este. Sin embargo, Esta medida de nuevo apuntaba a una empresa muy pequeña, dejando en evidencia que esta facultad solo se ha usado con empresas que no representaban un riesgo de paralización del sistema. Las empresas, aun impopulares y operando de manera oportunista, conservaban un importante margen de negociación, debido a los riesgos de detención de la ciudad. Después de todo, "[...] la sanción demasiado extrema, demasiado draconiana, al final no son posibles de implementar" (Entrevista con exministro no 3 ).

Sin duda, esta problemática constituye una muestra del poder estructural de las empresas. El inmenso riesgo político de una paralización del servicio genera que la caducación de contratos solo se aplique de forma puntual y contra empresas pequeñas. Esta fuente del poder estructural es la que se desarrolla en el contexto específico de la operación e implementación de políticas regulatorias, y es distinta del poder estructural que se manifestó en la adjudicación del sistema, tal como se mostró en el apartado anterior. A pesar de que los grandes operadores también habían incumplido sus contratos 
en más de alguna ocasión, los instrumentos que se aplican contra ellos no implican una medida tan drástica como lo es la caducación. Esta imposibilidad de sancionar de forma efectiva constituía la mayor dificultad para avanzar en la reestructuración del sistema anhelada por el equipo del ministerio en la administración de Piñera, tal como lo expresa un actor del proceso:

“[...] un problema del diseño de Transantiago es que son zonas muy grandes, entonces si un operador dice "no, no voy a aceptar eso y me voy", tú te podías imaginar la dimensión del problema, te quedas con un área entera de Santiago, sin transporte público. ¡Es una tragedia!” (Entrevista con alto funcionario público $\mathrm{n}^{\circ} 1$ ).

Frente a esta situación, se elaboró un proyecto de ley que buscaba otorgar mayores atribuciones al ejecutivo para controlar y fiscalizar a las empresas operadoras, además de renegociar o caducar contratos en los casos que fuera necesario. La tramitación de la ley fue particularmente rápida, a pesar de que el gobierno no tuviera mayoría en el Congreso, con su promulgación en enero de 2011 (ley 20.504). En este proceso nuevamente no se registran acciones concertadas por parte de los operadores para intentar influir sobre el proceso. La ley que se estaba gestando, al entregar mayores atribuciones a las autoridades, iba en claro desmedro de los transportistas, puesto que estos últimos estarían afectos a un mayor control. Nuevamente, no aparece evidencia de un ejercicio del poder instrumental por parte de las empresas transportistas.

Tras un cambio de gabinete en el año 2011, asume como ministro de la cartera Pedro Pablo Errázuriz. Con las nuevas facultades otorgadas por la ley 20.504, Errázuriz renegoció hasta obtener el año 2012 nuevos contratos, en los cuales se elimina la noción de alimentadores y troncales, asignando unidades de negocio específicas a los operadores, y traspasando la responsabilidad respecto a la evasión. El proceso empezó con reuniones entre el ministerio y las empresas, las que en primera instancia no lograron mayores resultados. Lo anterior, gatilló la necesidad de fortalecer los equipos del ministerio, para poner fin a cierta asimetría al momento de las negociaciones (Entrevista con ex ministro $\mathrm{n}^{\circ} 2$ ). También la ley 20.504 cumplió un rol fundamental para llevar a cabo el proceso de negociación ya que, de acuerdo con lo mencionado por una entrevistada, "con la herramienta en la mano, era como negociar con la pistola arriba de la mesa, sino no había ningún incentivo para que los operadores quisieran cambiar los contratos. ¡Si tenían la demanda garantizada!” (Entrevista con alto funcionario público n 1 ).

Errázuriz optó por realizar negociaciones por separado. Llegó primero a un acuerdo con tres empresas grandes, Vule, Metbus, y Subus, a las cuales se les ofreció escoger uno de los alimentadores a reasignar. Luego, el resto de los operadores tuvo que seguir la dinámica. Otra vez mostraron falta de cohesión. En esta oportunidad, el Estado, que se había reforzado para la ocasión, pudo acercarse más a sus preferencias generando una presión importante sobre los empresarios, quienes tuvieron que ceder parte de los beneficios que habían obtenido en los contratos originales. Así, la autoridad logró generar ciertos cambios en el funcionamiento del sistema que afectaron negativamente a los empresarios, dando cuenta de sus capacidades en el ámbito técnico y en la negociación política (Centeno, 2002; Peters, 1996).

En conclusión, en el marco del Transantiago, las relaciones que se desarrollan entre actores públicos y privados en la implementación de la política regulatoria generan que las autoridades puedan imponer parcialmente sus preferencias. Para explicar esto, la evidencia empírica arroja elementos que respaldan la hipótesis del poder estructural, ya que no hay mayores indicios de poder empresarial instrumental, ni tampoco de una debilidad de los actores públicos. En suma, en esta etapa se logra visibilizar la manera cómo actúa el poder estructural. Si bien la autoridad avanza en medidas regulatorias más 
duras para las empresas, éstas no se aplican con toda la firmeza que la capacidad estatal existente les permite. La explicación última de este fenómeno radica en que las decisiones de los policy-makers en este ámbito se encuentran constreñidas por las expectativas que tienen sobre el comportamiento de los actores privados (Fairfield, 2015b), y sobre el funcionamiento del sistema mismo. La aplicación de sanciones severas por parte de los ministros no era una opción viable a pesar de contar con todos los elementos a su favor, ya que no se podía correr el riesgo de que el servicio se interrumpiera.

Al igual que en la etapa de adjudicación, la implementación de la política regulatoria está constreñida fuertemente por el poder estructural de los transportistas. Sin embargo, el tipo de poder entre una y otra etapa varía considerablemente. Mientras en la licitación el Estado tenía un interés central en atraer oferentes y potenciar la inversión, en la implementación hay una preocupación por el riesgo de quiebra de las empresas, lo que significaría una paralización del servicio. Sin duda, para los actores estatales, este era un escenario que se debía evitar a toda costa. A pesar de su atomización y debilidad, los microbuseros no son tan afectados por los cambios que establece la autoridad, por el poder estructural propio en el ámbito de las concesiones.

\subsection{Entre quiebres y acuerdos: la (fracasada) construcción del poder instrumental de los empresarios transportistas}

En los apartados anteriores se han podido mostrar las capacidades técnicas y políticas que han expresado las autoridades en materia de regulación de transportes, así como también del poder estructural que han desarrollado los transportistas en relación con la política regulatoria bajo estudio. Sin embargo, llegado este punto es necesario referirse a los intentos de construcción de poder instrumental por parte de estos mismos empresarios vinculados al Transantiago, que son partes de la hipótesis del estudio. Específicamente, se ha documentado que los empresarios han generado intentos por construir cohesión entre ellos, lo que constituye uno de los atributos tradicionales del poder instrumental (Álamos, 2017).

Después de las negociaciones por separado con Errázuriz, los empresarios reaccionaron con la constitución en diciembre de 2013 de la Asociación de Concesionarias del Transporte Urbano de Superficie (ACTUS), gremio compuesto por la mayor parte de los operadores del Transantiago. Este referente buscaba representar a los concesionarios frente a instituciones públicas y privadas, teniendo un equipo técnico capaz de colaborar en las soluciones al Transantiago. Por primera vez en 6 años de funcionamiento, las empresas operadoras colaboraron formalmente para actuar de forma cohesionada frente a la autoridad. En la persecución de un mayor poder instrumental, la organización estableció también ciertos vínculos informales con el sector público, a través del reclutamiento de personeros políticos donde destaca la incorporación de Víctor Barrueto, ex diputado e Intendente de la Región Metropolitana, como director Ejecutivo. Además, se intentó construir un canal de cooperación técnica con el ministerio, materializado en estudios sobre Transantiago que fueron presentados a la autoridad, además de participar en sesiones de comisiones de la cámara de diputados, en las materias de su competencia.

Amparado en las facultades que proporcionaba la ley 20.504, el año 2015, Andrés Gómez-Lobo, ministro de transportes de la ahora presidenta Michelle Bachelet, empezó a reasignar recorridos, teniendo como principales afectados a operadoras grandes, de capitales extranjeros. Esta reasignación de recorridos le permitió a la autoridad oponerse a las empresas grandes sin exponerse a que se manifieste su poder estructural. En otros términos, constituye un recurso más accesible que la caducación, que en caso de las empresas grandes es una alternativa no factible, por la ausencia de servicios de respaldo, tal como se ha argumentado anteriormente. No obstante, la aplicación de esta nueva estrategia “[...] 
tensionó mucho las relaciones entre los operadores chilenos y colombianos" (Entrevista con dirigente empresarial $n^{\circ} 3$ ), teniendo implicancias en ACTUS y el curso de acción futuro de las operadoras, que ya estaban empezando a proyectarse hacia la licitación de la mitad de las unidades de negocio prevista para el año 2018. Así, a fines de 2015, las operadoras deciden disolver la organización producto de las mismas tensiones existentes a la interna, debido a que "[... ] las operadoras no soportaban a AlsaciaExpress, así de simple. O sea, no había una comunión de intereses" (Entrevista con experto no 1). Las estrategias de las empresas frente a la autoridad volvieron a divergir.

En el caso particular de Alsacia-Express, la compañía enfrentó una degradación de su situación financiera. La actualidad del sistema pasó a ser marcada por la búsqueda de una reestructuración de su deuda y riesgos de quiebra. En la perspectiva del poder empresarial, no deja de ser relevante el manejo político que se hizo del riesgo de la quiebra. En efecto, las empresas Subus y luego Alsacia Express pasaron a solicitarle directamente al ministro nuevas modificaciones contractuales, aludiendo que sin ellas podrían quebrar, y por lo tanto dejar importantes zonas de la ciudad sin servicio de transporte. También amenazaron con recurrir a la justicia internacional, en este caso el Centro Internacional de Arreglo de Diferencias relativas a Inversiones (CIADI). La autoridad no cedió a esta presión y finalmente la empresa Alsacia-Express levantó una demanda contra el Estado chileno ante el CIADI por "[...] incumplimiento de los contratos de concesión" (Diario Financiero, 2017) que se encuentra en tramitación.

Este episodio ilustra las tensiones que se pueden llegar a producir en una relación regulatoria, pero también confirma la forma en que los aspectos financieros (endeudamiento) y legales (disposiciones de los contratos) deben analizarse desde un punto de vista político, en el marco de una relación conflictiva. En término analítico, queda en evidencia la debilidad del poder instrumental de las empresas, con el fracaso de la construcción de un actor capaz de pesar en la discusión pública sobre el transporte. Aun así, el quiebre entre los operadores evidencia la existencia de estrategias alternativas. Algunos operadores optaron por una estrategia particularmente conflictiva, cuando otros, en la línea de las ambiciones de ACTUS, aunque sin la misma unión, siguieron persiguiendo estrategias de construcción de relación a largo plazo con la autoridad.

\subsection{Otra vez el poder estructural: nuevo proceso de licitación y reestructuración de la relación regulación-poder empresarial}

A casi 10 años del inicio del sistema, se comenzó a plantear el proceso de relicitación de la mitad de las unidades de negocio. Desde el punto de vista analítico, este escenario cambia nuevamente la evaluación de la relación entre regulación y poder empresarial. En el nuevo proceso de adjudicación, se hace muy fuerte el poder estructural clásico. A diferencia de la preocupación por el riesgo de quiebra que se expresaba en el apartado anterior, en esta etapa las autoridades vuelven a tener como principal preocupación lograr atraer inversionistas.

En principio, la autoridad quiso realizar un proceso licitatorio distinto al primero, incorporando por ejemplo una etapa temprana de consulta ciudadana, que buscaba identificar cuáles eran las preocupaciones de los usuarios. Empero, en este nuevo proceso se repitió el desafío de atraer a oferentes para la licitación. Con este propósito, se organizaron roadshows para presentar el proyecto. Para estos eventos se convocaron a operadores de transporte, pero el foco principal estaba en instituciones financieras, particularmente extranjeras, para que aportaran financiamiento a los oferentes. Esto pone de relieve el peso de los constreñimientos financieros sobre la relación que se establece en el marco de este proceso de regulación. 
El proceso de relicitación recién descrito, nos retrotrae a los procesos licitatorios iniciados a comienzos del 2000. El poder estructural tradicional que era observado durante la primera licitación del Transantiago, vuelve a surgir con fuerza ante este nuevo panorama. Empíricamente, esto es evidenciado a través de las condiciones establecidas en los contratos de concesión para esta nueva licitación. En tal sentido, dos son los principales elementos que permitan sopesar la importancia del poder estructural: establecer criterios laxos para atraer a nuevas empresas y generar condiciones para asegurar la participación de inversionistas que permitan la solvencia financiera del sistema.

Respecto a esto último, el subsecretario de la época, Carlos Melo, había anunciado que los contratos podrían tener una duración menor a los 10 años de las primeras concesiones. Sin embargo, se optó por mantener esta misma cantidad, la que, según la ministra Tapia, "[...] es un periodo interesante para los inversionistas" (Pulso, 2017), ya que entrega mayor seguridad en cuanto a la posibilidad de recuperar la inversión inicial. Se aprecia ahí el poder estructural del sector financiero, y cómo la autoridad política tiene que tomarlo en consideración, en general en desmedro de sus preferencias, en este caso por un proceso de contractualización más ágil. Aun así, las ofertas presentadas en la licitación son pocas, con solo tres operadores extranjeros nuevos que presentaron oferta. Poco antes de ser adjudicada, la licitación fue suspendida por el Tribunal de Defensa de la Libre Competencia, que acogió una demanda presentada por una pequeña empresa de transporte que alude a la existencia de barreras de entrada. Algunas semanas después, la nueva Ministra Hutt, parte del recién estrenado gobierno de Sebastián Piñera, anuncia la cancelación del proceso de relicitación, y su postergación hasta ser reformulado. Aun sin conocer el destino final de este proceso, esta tercera etapa deja de manifiesto las dificultades para el actor público de contrarrestar el poder estructural del sector privado, aun cuando este dispone de poco poder instrumental.

Para sintetizar la narrativa, presentamos la evaluación de las tres hipótesis de estudio.

\section{CUADRO 3 EVALUACIÓN DE LAS HIPÓTESIS DE ESTUDIO}

\begin{tabular}{|c|c|c|}
\hline Hipótesis & Evidencia & Evaluación. \\
\hline $\begin{array}{l}\text { H1a. Poder } \\
\text { instrumental de } \\
\text { los operadores }\end{array}$ & $\begin{array}{l}\text { El intento de los operadores de construir cohesión } \\
\text { fracasa. No tienen experticia técnica solicitada por la } \\
\text { autoridad, ni gozan de una buena imagen pública. }\end{array}$ & $\begin{array}{l}\text { Los operadores muestran escaso poder } \\
\text { instrumental, por lo que no puede constituir el } \\
\text { factor que explique su capacidad de resistir a las } \\
\text { preferencias del actor público. }\end{array}$ \\
\hline $\begin{array}{l}\text { H1b. Poder } \\
\text { estructural de } \\
\text { los operadores }\end{array}$ & $\begin{array}{c}\text { Tanto en la licitación inicial como en la relicitación, } \\
\text { la preocupación principal de la autoridad es hacer } \\
\text { la concesión atractiva para los inversionistas, en } \\
\text { particular financieros. Durante la concesión, el } \\
\text { riesgo de quiebra y en general el riesgo político de } \\
\text { la suspensión del servicio es un instrumento de } \\
\text { presión muy eficaz. }\end{array}$ & $\begin{array}{c}\text { El poder estructural de los actores privados - } \\
\text { operadores e instituciones financieras - que } \\
\text { participan directa o indirectamente del sistema } \\
\text { es muy importante. Constituye el principal factor } \\
\text { explicativo de las dificultades para el ejecutivo de } \\
\text { llevar a cabo las transformaciones que tienen su } \\
\text { preferencia. }\end{array}$ \\
\hline $\begin{array}{l}\text { H2. Deficiencias } \\
\text { de los } \\
\text { organismos } \\
\text { públicos y la } \\
\text { autoridad }\end{array}$ & $\begin{array}{l}\text { No aparecen mayores deficiencias de los } \\
\text { organismos públicos. Las autoridades son } \\
\text { competentes y tienen respaldo político, tanto de los } \\
\text { presidentes de turno como de los parlamentarios, } \\
\text { que mostraron una sorprendente unanimidad. Los } \\
\text { problemas financieros del sistema no son decisivos } \\
\text { en la relación con los operadores. }\end{array}$ & $\begin{array}{l}\text { Se rechaza la hipótesis alternativa como factor } \\
\text { explicativo central del proceso. Si las autoridades } \\
\text { no pueden imponer sus preferencias, no es por } \\
\text { deficiencias propias. }\end{array}$ \\
\hline
\end{tabular}

Fuente: Elaborado por los autores. 


\section{CONCLUSIÓN}

A través del estudio de caso de la regulación del transporte público urbano de Santiago, se ha demostrado la importancia de la incorporación de los aportes conceptuales de la teoría del poder empresarial para una comprensión completa de los procesos regulatorios. El Transantiago constituye una de las políticas más cuestionadas por la ciudadanía en las últimas décadas. La mala calidad del servicio que se hizo notar desde su primer día de funcionamiento animó los constantes cambios contractuales graduales que se han desarrollado en más de una década de funcionamiento del sistema. En este contexto, las relaciones entre los empresarios transportistas y las autoridades políticas, a través de los distintos instrumentos de política pública en materia de regulación, se vuelven fundamentales para comprender la dificultad para modificar la regulación y el servicio mismo.

En definitiva, el estudio del caso de Transantiago valida la premisa que motivó este artículo. Si bien la práctica regulatoria se relaciona con el diseño del contrato, las variables políticas son fundamentales para comprender las dinámicas y los resultados que se obtienen. Por lo tanto, es de esperar que cualquier renegociación de concesión sea la oportunidad para que se desarrolle un juego de poder entre los distintos actores involucrados. En esta configuración, las herramientas analíticas de la economía política permiten una acabada comprensión de los cursos de acción adoptados por las distintas partes. Así, se concluye que las autoridades no pudieron imponer sus preferencias políticas a las empresas operadoras del sistema, no porque estas, como a veces se cree, hayan tenido una fuerte capacidad de lobby. En este caso, el resultado no se generó ni por acciones deliberadas de las concesionarias relativamente débiles en materia de poder instrumental, ni por falta de capacidad estatal. Más bien, la explicación se encuentra en el ejercicio de un poder estructural que se establece desde los orígenes del sistema, y que se encuentra estrechamente ligado a los requerimientos de financiamiento. Así, el poder estructural de los empresarios es fundamental para explicar estas dinámicas en las políticas regulatorias del sistema de transporte urbano de Santiago. Esto crea las condiciones para una trayectoria posterior de "dependencia al sendero", que se prolonga hasta la actualidad.

Adicionalmente, hemos podido determinar que este poder estructural se expresa de dos formas, dependiendo de la fase en que se encuentre la política. Por un lado, en el proceso de adjudicación actúa el poder estructural en su forma tradicional, es decir, los policy-makers toman decisiones en el ámbito político resguardo y promoviendo la inversión privada (Fairfield, 2015a). Por otro lado, en la fase de adjudicación, el poder estructural se manifiesta como el constreñimiento de las autoridades reguladoras, producto de las expectativas que tengan sobre la posible quiebra de las empresas y la consiguiente discontinuidad en el servicio que sus decisiones en materia de regulación, así como sus sanciones, podrían acarrear.

Visto desde este ángulo, el caso de Transantiago es un caso típico de contractualización, por lo que es de esperar que estas dinámicas, y en particular esta fuerza del poder estructural, se manifiesten también en otras situaciones de colaboración público-privada. Teniendo en cuenta estos elementos, el estudio acá realizado representa una contribución en a lo menos dos sentidos. Por una parte, incita al estudio de las políticas regulatorias desde perspectivas analíticas distintas a las utilizadas tradicionalmente en disciplinas como la gestión pública y el derecho. La capacidad heurística de las corrientes teóricas acá utilizadas demuestra la relevancia de incorporar este tipo de análisis en procesos políticos complejos, donde los actores públicos y privados desarrollan distintos tipos de interacciones en función de sus objetivos, muchas veces contrapuestos. Por otro lado, la investigación también ha logrado establecer aprendizajes valiosos para los procesos de contractualización y para las políticas regulatorias en general. Así, queda en evidencia la importancia de sumar a las herramientas tradicionales de los diseños de contrato estas preocupaciones políticas en cuanto a la aplicabilidad real de las sanciones, fuertemente limitada por el poder estructural de las empresas. 


\section{REFERENCIAS}

Álamos, V. (2017). Poder empresarial y políticas públicas: el caso de las reformas laborales en el Chile post dictadura. (Tesis de Magíster). Universidad de Chile, Santiago, Chile.

Athias, L., \& Saussier, S. (2007). Contractual flexibility or rigidity for public private partnerships? Theory and evidence from infrastructure concession contracts. Munich, Alemania: University Library of Munich.

Baldwin, R., Cave, M., \& Lodge, M. (2010). The Oxford Handbook of Regulation. Nueva York, Estados Unidos: Oxford University Press.

Bell, S., \& Hindmoor, A. (2013). The Structural Power of Business and the Power of Ideas: The Strange Case of the Australian Mining Tax. New Political Economy, 19(3), 470-486.

Besley, T., \& Persson, T. (2009). The origins of state capacity: Property rights, taxation, and politics. American Economic Review, 99(4), 1218-1244.

Besley, T., \& Persson, T. (2008). Wars and state capacity. Journal of the European Economic Association, 6(23), 522-530.

Bennet, A., \& Checkel, J. (2015). Process Tracing in the Social Sciences. From Metaphor to Analytic Tool. Cambridge, Reino Unido: Cambridge University Press.

Bhagat, G. (2017). Public Procurement: a Competition Perspective. Indian Journal of Public Administration, 63(2), 176-186.

Bitrán, E., Nieto-Parra, S., \& Robledo, J. (2013). Opening the black box of contract renegotiations: An Analysis of Road Concessions in Chile, Perú and Colombia. (Working Paper, 317). Paris, Francia: OECD Development Centre.

Bovaird, T. (2006). Developing new forms of partnership with the 'market'in the procurement of public services. Public administration, 84(1), 81-102.

Bril, T., Maillet, A., \& Mayaux, P.-L. (2017). Process tracing: inducción, deducción e inferencia causal. Revista de Ciencia Política, 37(3), 659-684.

Centeno, M. A. (2002). Blood and Debt: War and the Nation-State in Latin America. Pennsylvania, USA: Penn State Press.
Christensen, T., \& Lægreid, P. (Eds.). (2006). Autonomy and regulation: Coping with agencies in the modern state. Massachusetts, USA: Edward Elgar Publishing.

Cingolani, L. (2013). The State of State Capacity: a review of concepts, evidence and measures. (MERIT Working Papers, n. 53). Ámsterdam, The Netherlands: Institutions, Governance and Longterm Economic Growth.

Córtazar, R. (2015). Transantiago: 10 claves para enfrentar crisis. Santiago, Chile: Uqbar Editores.

Culpepper, P. (2011). Quiet Politics and Business Power: Corporate Control in Europe and Japan. New York, USA: Cambridge University Press.

Culpepper, P. (2015). Structural power and political science in the post-crisis era. Business and Politics, 17(3), 391-409.

Dente, B., \& Subirats, J. (2014). Decisiones públicasAnálisis y estudio de los procesos de decisión en políticas públicas. Barcelona, España: Ariel.

Engel, E., Fischer, R., \& Galetovic, A. (2010). The economics of infrastructure finance: Publicprivate partnerships versus public provision. EIB papers, 15(1), 40-69.

Espacio Público. (2017, enero). Transantiago: nueva licitación que abre oportunidades. (Informe de políticas públicas, 11). Santiago, Chile: Fundación Espacio Público.

Evans, P. (1996). El Estado como problema y como solución. Desarrollo Económico, 35(140), 529-562.

Fairfield, T. (2015a). Structural power in comparative political economy: perspectives from policy formulation in Latin America. Business and Politics, 17(3), 411-441.

Fairfield, T. (2015b). Private Wealth and Public Revenue in Latin America. Business power and tax politics. New York, USA: Cambridge University Press.

Figueroa, C., \& Forray, R. (2011). Transantiago: Convergencias y divergencias entre la vocación de servicio público y la racionalidad técnico-económica del sistema. La fabrique du mouvement.

Gilardi, F., \& Maggetti, M. (2011). The independence of regulatory authorities. In Levi-Faur, D. (Ed.). Handbook on the politics of regulation. Massachusetts, USA: Edward Elgar Publishing. 
Girth, A. (2012). A closer look at contract accountability: Exploring the determinants of sanctions for unsatisfactory contract performance. Journal of Public Administration Research and Theory, 24(2), 317-348.

Goertz, G.; \& Mahoney, J. (2012). A tale of two cultures: Qualitative and Quantitative Research in the Social Sciences. New Jersey, USA: Princeton University Press.

Gómez-Lobo, A. (2007). Transantiago: una reforma en panne. TIPS, Trabajos de Investigación en Políticas Públicas, 4, 1-14.

Hacker, J., \& Pierson, P. (2002). Business power and social policy: Employers and the formation of the American welfare state. Politics \& Society, 30(2), $277-325$

Jordana, J., \& Levi-Faur, D. (2005). The diffusion of regulatory capitalism in Latin America: Sectoral and national channels in the making of a new order. The Annals of the American Academy of Political and Social Science, 598(1), 102-124.

Lindblom, C. (1982). The Market as Prison. The Journal of Politics, 44(2), 324-333.

Maillet, A. (2008). La gestación del Transantiago en el discurso público: hacia un análisis de políticas públicas desde la perspectiva cognitivista. In M. De Cea, P. Díaz, \& G. Kerneur. (Eds.), Chile: de país modelado a país modelo. Una mirada sobre la política, lo social y la economía. Santiago, Chile: Gresch.

Maillet, A. (2015). Más allá del "modelo" chileno: una aproximación multi-sectorial a las relaciones Estadomercado. Revista de Sociología E Política, 55, 53-73.

Mardones, R. (2008). Chile: Transantiago recargado. Revista de Ciencia Politica, 28(1), 103-119.

Menezes, F., Pitchford, R., \& Wait, A. (2003). Tendering and bidding for access: A regulator's guide to auctions. Australian Journal of Management, 28(3), $345-370$
Ministerio de Transportes y Telecomuinicaciones. (2003). Bases para la licitación del estudio. Santiago, Chile: MTT.

Mukhopadhyay, B. (2011). Evaluating public procurement. Review of Market Integration, 3(1), 21-68.

Olavarría, M. (2013). De la formulación a la implementación del transantiago. Análisis del Proceso Político de una Política Pública. Revista Gestión Y Politica Pública, 22(2), 355-400.

Osborne, D., \& Gaebler, T. (1992). Reinventing government: How the entrepreneurial spirit is transforming government. Reading Mass. Adison Wesley Public Comp, 33(6), 97-99.

Peters, G. (1996). The policy capacity of government. Ottawa, Canada: Canadian Centre for Management Development.

Ramírez, P., \& Minay, S. (2007). El recorte que mató al Transantiago. Santiago, Chile: CIPER. Recuperado de https://ciperchile.cl/2007/11/04/el-recorte-quemato-al-transantiago/

Skocpol, T. (1985). Bringing the state back in: strategies of analysis in current research. En P. Evans, D. Rueschemayer, \& T. Skocpol. (Eds.), Bringing the State Back in. Cambridge, Reino Unido: Cambridge University Press.

Stigler, G. (1971). The theory of economic regulation. The Bell journal of economics and management science, 2(1), 3-21.

Trampusch, C., \& Palier, B. (2016). Between X and Y: how process tracing contributes to opening the black box of causality. New Political Economy, 21(5), 437-454.

Ureta, S. (2015). Assembling Humans: Transport Infrastructures and the Dream of a World-Class City in Chile. Massachusetts, USA: MIT press.

Wettenhall, R. (2003). The rhetoric and reality of public-private partnerships. Public Organization Review, 3(1), 77-107. 
RAP | Regulación y poder empresarial: el caso del transporte público en Santiago (2007-2017)

\section{Antoine Maillet}

https://orcid.org/0000-0002-4607-3964

Doctor en Ciencia Política; Profesor Asistente del Instituto de Asuntos Públicos de la Universidad de Chile. E-mail: antoinemaillet@iap.uchile.cl

\section{Sebastián Carrasco}

$$
\text { https://orcid.org/0000-0003-0081-2909 }
$$

Estudiante del Doctorado en Políticas Públicas de la Universidad Mayor; Magíster en Ciencia Política por el Instituto de Asuntos Públicos de la Universidad de Chile. E-mail: sebastian.carrasco.s@ug.uchile.cl 